\title{
Towards optimal activation of balancing energy to minimize regulation from neighboring control areas
}

\author{
Zane Broka, Karlis Baltputnis, Antans Sauhats \\ Institute of Power Engineering \\ Riga Technical University \\ Riga, Latvia \\ zane.broka@rtu.lv, karlis.baltputnis@rtu.lv, \\ sauhatas@eef.rtu.lv
}

\begin{abstract}
The paper presents practical research based on a reallife case study of the Baltic power system for optimal activation of manual frequency restoration reserves. A software tool for the determination of the activation volume and time schedule is developed to facilitate the decision-making process of the transmission system operator in balancing of the power system within a coordinated balancing area. The study is important in light of the ongoing integration of balancing markets within the European Union and the subsequent need to develop an activation optimization function. Recently, regular balancing needs of the Baltic countries were covered by the neighboring Russian power system. The motivation for this study was moving towards local regulation as much as possible for energy dependence related and economic reasons. Moreover, development of the activation optimization function has served as one of the first steps towards creating a fully automated balancing system.
\end{abstract}

Index Terms--balancing, frequency restoration reserves, optimization, power system control.

\section{INTRODUCTION}

The European Commission Regulation on electricity balancing aims to foster formation of integrated balancing markets to enable cost-efficient and reliable exchange of balancing services among the European countries [1]. To implement this, relevant ICT tools need to be developed, as until now the balancing of power systems is still often human operator dependent. Balancing bids for activation should be selected from merit order lists containing bids of standardized balancing products. This paper is focused on the recently launched common Baltic balancing market by the Latvian, Lithuanian and Estonian transmission system operators (TSOs) with the overarching objective to develop a common NordicBaltic balancing market for manually activated frequency restoration reserves (mFRR) [2].

The Baltic power system has some distinct characteristics due to its synchronous operation with the Integrated/Unified Power System (IPS/UPS) of Russia and Belarus. The Russian power system provides primary power reserves for frequency regulation and secure system operation within the BRELL

\author{
Gatis Junghans, Liga Sadovica, Valentins Lavrinovics \\ Augstsprieguma tīkls AS \\ Riga, Latvia \\ gatis.junghans@ast.lv, liga.sadovica@ast.lv, \\ valentins.lavrinovics@ast.lv
}

(Belarus, Russia, Estonia, Latvia and Lithuania) ring [3]. As of 2018, the TSOs of Latvia, Estonia and Lithuania (Augstsprieguma tīkls AS, Elering and Litgrid) have established a common Baltic balancing market within which the three countries are able to share balancing energy. However, the remaining not netted imbalance is settled by an Open Balance Provider (OBP) (Fig. 1) which provides balancing energy from the Russian power system via seven transmission lines. The imbalance settlement period (ISP) currently is one hour, and the not netted imbalance with the OBP is defined as the total Baltic Area Control Error (ACE), or the overall system imbalance. It is calculated as the difference between the scheduled and the actual power flow each minute, integrated over the whole ISP to obtain the final ACE in MWh. Thus, the payment for the Baltic ACE covers the cost of the Russian frequency control service [4].

Formally, the three Baltic countries are required to keep their imbalance within certain limits $( \pm 30 \mathrm{MWh} / \mathrm{h}$ for Estonia and Latvia each and $\pm 50 \mathrm{MWh} / \mathrm{h}$ for Lithuania [2]). In practice, until the end of 2017 minor imbalances were handled by the

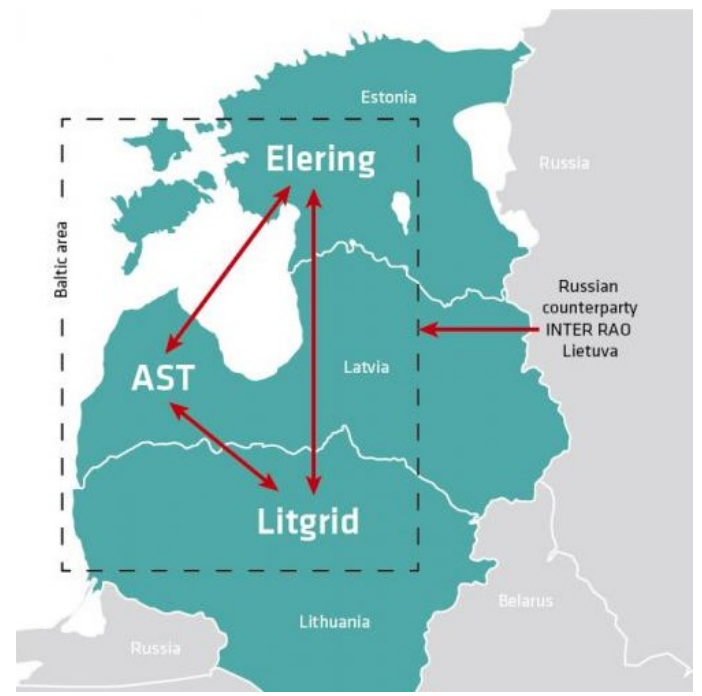

Figure 1. Imbalance netting of the Baltic TSOs under the Open Balance Agreement [4]

The work presented in this paper has been co-financed by the National Research Program LATENERGI (2014-2017). 
imports from Russia on a regular basis while local activations were used to cover larger imbalances on comparatively rare occasions. As a result, the ACE energy constituted a major part of the total Baltic balancing energy and contributed a significant share of balancing costs in the Baltic markets (e.g., some $40 \%$ of total balancing costs in Latvia in 2014 [4]). Handling ACE with energy from the Russian power system is costly due to the specific pricing policy employed by the OBP: a low sell price fixed at $5 € / \mathrm{MWh}$ and a high buy price which may exceed $100 € / M W h$ (data of 2015) [4]. For context, the average dayahead market electricity price in the three Nord Pool bidding areas of the Baltic countries was 35.23 €/MWh in 2016.

The aforementioned considerations along with the political decision to cease synchronous operation with the IPS/UPS by 2025 [5] have encouraged the Baltic TSOs to develop a Baltic balancing IT system with the primary function to ensure sustainable physical cross-border balancing. This system facilitates sharing balancing energy among the three countries with the aim to increase reliance on mFRR provided by local producers, the bids of which are included in the Baltic common merit order list (CMOL). It is expected to reduce the overall balancing costs incurred by the three Baltic TSOs, while contributing to the energy independence of Latvia, Estonia and Lithuania.

One of the building blocks of the common balancing system is the Activation Optimization Function (AOF). As stipulated in the guidelines [1] developed by ENTSO-E, the AOF determines the most efficient activation of the incoming balancing request while respecting some capacity and operational restrictions. The Baltic TSOs intend to implement the AOF as an automatic algorithm the main inputs to which are the available bids from the CMOL (considering transmission constraints) and activation volume proposal [2], the latter being the focus of this paper. Specifically, it implies an algorithm for determination of optimal activation volume of balancing reserves along with a time schedule based on the historic ACE data with minute resolution and the current ACE forecast. It is meant to support the decision-making by the dispatch operator of the transmission system, which thus serves as the first step towards building a fully automatic system for the activation of balancing reserves.

As of now, the ordering of the balancing energy is left solely to the dispatch operator with a very short timeframe for decision-making. Combining the time restriction with the large number of variable and uncertain parameters of the power system, it stands to reason that an automated tool should provide operational advantages. However, the significant hands-on experience of dispatch operators, which is challenging if not outright impossible to translate into an automated algorithm [6], cannot be dismissed or ignored. Thus, one of the tasks of this study has been to investigate the pros and cons of automated vs manual regulation activation.

Until now, only a few scientific papers refer to the AOF introduced in the recently established guidelines on electricity balancing [1]. One of the studies [7] proposes balancing optimization based on stochastic unit commitment principles using imbalance forecast scenarios. The objective of optimization in [7] is to minimize expected activation costs, which is demonstrated using Norwegian imbalance and market data. As a result, bid activation schedules are proposed. The imbalance forecasts are generated from probability distributions of historical data series, and balancing activation bids are created based on prices and volumes in the Norwegian balancing energy market. Utilization of both mFRR and aFRR is considered.

Case studies of optimal scheduling of ancillary services (AS) for the Czech Republic are presented in [8] and [9]. In [8], five different types of AS used by the Czech TSO are considered to minimize the cost of balancing. Power imbalances and the resulting ACE is obtained from MonteCarlo simulations to imitate the random behavior of the power system, while the AS prices are assumed as estimated by experts due to the complexities related to modelling the entire AS market. In [9], an evolutionary algorithm for cost-optimal dispatch of AS is used and regulation reserves are modeled for a 6-hour horizon. Comparison of the historical vs optimized activations shows that the ACE and regulation energy costs decrease in the latter case.

\section{METHOD}

Within this study, we have developed a software tool for deriving optimal activation parameters of $\mathrm{mFRR}$ for balancing of the Baltic power system. The main objective of the algorithm implemented is to identify close to optimal regulation parameters and to evaluate the performance of balancing operations carried out in accordance with these parameters. The optimization problem is formulated and solved in MATLAB to take advantage of its data processing abilities and solvers. MS Excel is used for input and output due to its user-friendly interface.

\section{A. Overall Structure of the Algorithm}

The algorithm operates under the assumption that the mFRR should be activated several times within the given ISP (in our case study, we assume no more than five activations within an ISP of one hour). It calculates the amount of up or down balancing power which ought to be activated based on three main parameters which serve as optimization variables within the algorithm: act time - the time of activation (minutes from the beginning of each ISP), frc perc - the percentage of the ACE forecast to be regulated against and ign thr - the ignorance threshold (the minimum value of the $\mathrm{ACE}$ forecast for regulation to be activated).

The most important parts of the algorithm are as follows:

1) reading input data of ACE forecast and user-selected settings for regulation and optimization, such as ISP duration, max number of activations per ISP, preparation time before mFRR activation, ramping rate, minimum interval between two subsequent activations and bounds on optimization variables (i.e., regulation parameters);

2) preprocessing of input data series by dividing into training and testing subsets and filtering out hours with "odd" behavior which might negatively influence the overall performance of the optimization solver;

3) the processed data and constraints are then used to formulate the optimization problem to be passed to the solver which, in its turn, evaluates the objective function by moving 
though the search space. The objective function contains all the decision logic for mFRR activation as performed by the TSO. The resulting optimization problem is non-smooth, and its convexity cannot be established analytically for a general case. Thereby, the use of gradient-based solvers is not recommended, and rather global search techniques must be employed. For this study, we employ the MATLAB Pattern Search function [10] with 50 randomly selected initial points. It should be noted that the results obtained are not guaranteed to be the global minima, but they can reasonably be expected to be sufficiently close to it and generally better than any arbitrarily selected regulation parameters;

4) once the solver has found the resulting close to optimum parameters for regulation, they are once more passed to the mFRR activation logic, but now with the testing dataset to evaluate the performance of the AOF algorithm with the optimized parameters under the conditions of new "unseen" ACE data.

\section{B. ACE Forecast}

In general, time series of ACE forecast that are provided as input data for optimization can be either a historical record of ACE forecasts or simulated time series created for simulation purposes. In our study, we used real-life historic data of 2016 with a minute resolution from the SCADA/EMS provided by the TSO. Since forecasting per se was out of scope of this study, we employed an already existing naïve ACE forecasting approach used by the TSO:

$$
A C E_{\text {forec. }}^{m}=\left(P_{\text {actual }}^{m} \cdot(M-m+1)+\sum_{k=1}^{m-1} P_{\text {actual }}^{k}-\sum_{k=1}^{M} P_{\text {plan }}^{k}\right) \cdot \frac{\Delta t}{60},
$$

where $m$ is the index of the particular minute, $M$ is the length of the ISP in minutes, $A C E_{\text {forec. }}^{m}$ is the forecast of the hourly ACE (MWh) at minute $m, P_{\text {actual }}^{m}$ is the actual power flow measured at minute $m$. The two summation operators calculate the total actual power flow $P_{\text {actual }}$ from the beginning of ISP till the previous minute, $m-1$, and the planned power flow, $P_{\text {plan }}$, for the whole ISP, where $k$ indexes through the time steps $\Delta t$. Since the time step, in our case, is equal to one minute, we divide the equation by 60 in order to obtain energy (ACE forecast) in MWh. The power flows here refer to the total scheduled Baltic power flow balance (after Nord Pool dayahead and intraday market clearing) and the actual Baltic balance. The results of the case study presented further on demonstrate overall good applicability of this ACE forecasting approach. Nevertheless, it is also one of the possible directions for improvement of the AOF algorithm in the future work. Some of the approaches suggesting a more sophisticated forecasting of system imbalance volumes are provided in [11] and [12].

\section{Objective Function}

The objective function to be minimized during the optimization is formulated as follows:

$w_{1 \mathrm{a}} \cdot \sum_{n=1}^{N} A C E_{+}^{n}+w_{1 \mathrm{~b}} \cdot \sum_{n=1}^{N}\left|A C E_{-}^{n}\right|+w_{2} \cdot \sum_{n=1}^{N}\left|E_{\text {suppl. }}^{n}\right|+w_{3} \cdot \sum_{n=1}^{N}\left|P_{\text {ord. }}^{n}\right|$, where $w_{1 \mathrm{a}}, w_{1 \mathrm{~b}}, w_{2}, w_{3}$ - weight coefficients for the various criteria of the multi-objective problem statement; $A C E_{+}^{n}-$ positive ACE, or net balancing energy exported to the Russian power system $(\mathrm{MWh} / \mathrm{h})$ during the ISP $n ; A C E_{-}^{n}-$ negative $\mathrm{ACE}$, or net balancing energy imported from the Russian power system $(\mathrm{MWh} / \mathrm{h})$ during the ISP $n ; E_{\text {suppl. }}^{n}-$ total provided balancing energy (MWh) during the ISP $n ; P_{\text {ord. }}^{n}$ - sum of the ordered regulation power (MW) during the ISP $n ; n, N$-index and number of the ISPs in the training dataset.

The regulation simulation logic is implemented in such a way that $A C E_{+}^{n}, A C E_{-}^{n}, E_{\text {suppl. }}^{n}$ and $P_{\text {ord. }}^{n}$ are functions of the optimization variables mentioned before: act_time, frc perc, ign_thr. The purpose of the weight coefficients is to give an option to adjust the focus on ACE minimization (positive and/or negative) versus utilized balancing energy and ordered balancing power. Sensitivity analysis on the influence of these coefficients is out of the scope for this paper but will be provided in future publications. For this study, the weight coefficients had fixed values of 1000, 1000, 10 and 1, respectively, which were chosen after a series of experiments. Thus, our primarily goal was to minimize the ACE at the end of each ISP, while also trying to efficiently reduce the amount of balancing energy used and the ordered regulation power.

\section{RESULTS AND DISCUSSION}

To obtain (close to) optimum mFRR activation parameters, the optimization procedure was applied to historical data of the year 2016 provided by the TSO which was divided into training and testing subsets. Data series of the ACE forecast (eq. 1) and the actual ACE with minute resolution was split into threemonth periods in an attempt to capture seasonal differences in data. This approach also reflects the intended use of the algorithm by the Baltic TSOs, namely, its application on historic data of one or a few months to obtain activation parameters which are then used to assist the operators in balancing the power system for upcoming periods (e.g., one or a few months). Regulation parameters were optimized on the training data set comprising the first $2 / 3$ of the whole preprocessed set. To test the performance of the optimized balancing schedule, the estimated parameters where applied on the testing data set (i.e., the remaining 1/3) to simulate all activities of regulation as performed by the TSO. Consequently, the results presented show the performance of regulation only for the testing set and allow us to evaluate the generalization ability of the optimization procedure.

The optimized activation parameters and resulting ACE for one of the data sets (July-September 2016) are shown in Table I. The resulting ACE after simulating the balancing activities according to the optimized schedule for the same time period is shown in Fig. 2. The ACE is noticeably reduced (up to 4.9 times) from $37.95 \mathrm{MWh} / \mathrm{h}$ before any regulation to $7.7-$ 12.5 MWh/h. The stacked chart allows to assess the efficiency of regulation in terms of the provided balancing energy and improvement of the ACE. The sum of the ACE after regulation and provided balancing energy is always more than the ACE without any regulation because of the ACE uncertainty which sometimes causes redundant orders (leading to cancellation of 
previous balancing orders or overregulation). In the data period shown in Fig. 2, the most efficient regulation happens when the max number of activations is 4 or 5 . Then, cancellation is needed for only $1.4 \%$ or $1.7 \%$ of the ordered energy respectively. Also, less balancing energy is used to reduce the ACE compared to cases with 2 or 3 activations. This is due to more gradual and cautious regulation which is possible with a larger number of activations within the ISP.

It is important to note that the average absolute error of the ACE forecast at the minute 45 was $6.34 \mathrm{MWh} / \mathrm{h}$ in Jul-Sep 2016 (Table I). This is very close to the lowest average ACE value achieved $(7.71 \mathrm{MWh} / \mathrm{h})$ which again demonstrates the efficiency of the proposed activation parameters when applied on the testing set.

In all the cases with 2 to 5 max activations, the last selected activation minute is 45 , which is the upper bound imposed during the optimization. This is due to the initially highly uncertain nature of the ACE forecast, the accuracy of which significantly increases towards the end of each ISP (Fig. 3). Since premature activation can lead to redundant orders for regulation and the subsequent cancellation of regulations that happen to be in the opposite direction, the algorithm evidently tends to postpone activations as long as possible.

Fig. 4 presents frequency distribution of the hourly ACE without and with regulation applied to the testing subset of JulSep 2016 dataset (509 hours in total). The ACE without regulation (blue bars) is moderately skewed to the right with an average value of $+21.9 \mathrm{MWh} / \mathrm{h}$. This can be explained by the behavior of balance responsible parties (BRP) who tend to prefer long rather than short positions because the potential financial risk for 'short' prices is inclined to be more extreme than for 'long' prices [4]. As a result, the Baltic countries in general sell more energy to the OBP than they buy.

After applying the optimized regulation parameters to the test set of Jul-Sep 2016, the average ACE decreases from $+21.9 \mathrm{MWh} / \mathrm{h}$ to $+2.5 \mathrm{MWh} / \mathrm{h}$ (Fig. 4 , orange bars). Thus, the noticeable positive bias of the ACE is almost eliminated. This

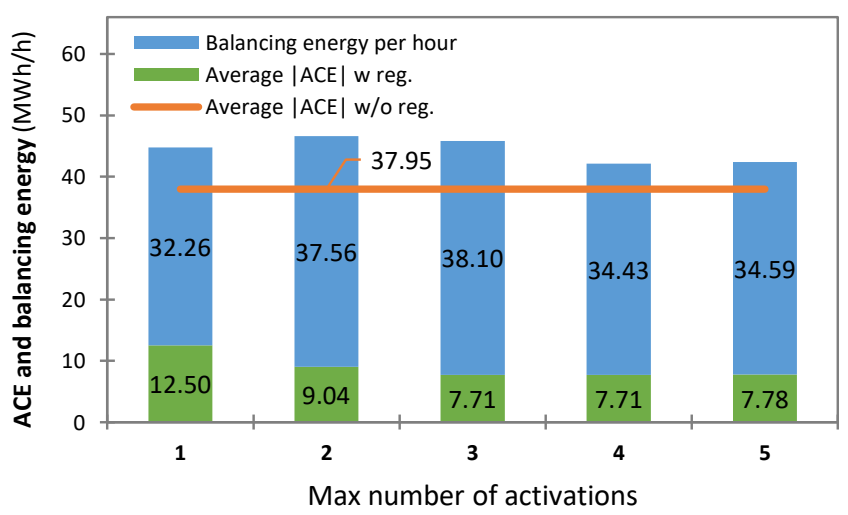

Figure 2. ACE w/o and with reg. and used balancing energy

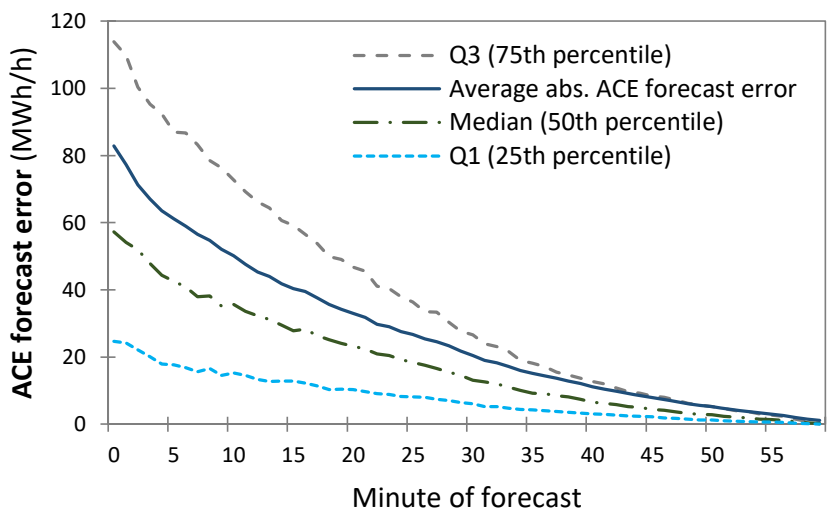

Figure 3. Statistics of the absolute ACE forecast error depending on the minute of forecasting within the ISP of one hour

clearly demonstrates not only a good performance of regulation with the optimized parameters but also the generalizability of the obtained parameters when applied to the testing data.

TABLE I.

OPTIMIZED REGULATION PARAMETERS AND RESUltiNG IMBALANCE

\begin{tabular}{|c|c|c|c|c|c|c|c|}
\hline \multicolumn{8}{|l|}{ Optimized reg. parameters } \\
\hline max number of activations & & & 1 & 2 & 3 & 4 & 5 \\
\hline activation minutes & & & 35 & $23-45$ & $22-34-45$ & $15-25-35-45$ & $5-15-25-35-45$ \\
\hline $\begin{array}{l}\text { volume of activation } \\
\text { relative to ACE forecast }\end{array}$ & $\%$ & & 90.5 & $75.4-97.4$ & $65.9-81.9-98.5$ & $50.1-50.7-80.6-93.7$ & $31.1-31.1-31.1-76.7-94.3$ \\
\hline ignorance threshold & MWh & & 10.0 & $22.1-10.0$ & $33.0-27.5-10.0$ & $192.0-107.0-31.0-10.0$ & $182.0-137.5-81.8-33.0-10.0$ \\
\hline Imbalance indicators & & $\begin{array}{c}\text { Without } \\
\text { local reg. }\end{array}$ & \multicolumn{5}{|c|}{ After local regulation with optimized parameters } \\
\hline Average $|\mathbf{A C E}|$ & $\mathrm{MWh} / \mathrm{h}$ & 37.95 & 12.50 & 9.04 & 7.71 & 7.71 & 7.78 \\
\hline Average ACE & $\mathrm{MWh} / \mathrm{h}$ & 21.85 & 4.42 & 3.63 & 2.47 & 2.73 & 2.55 \\
\hline Sum $|\mathrm{ACE}|$ & $\mathrm{MWh}$ & 19315 & 6364 & 4601 & 3924 & 3923 & 3961 \\
\hline Sum pos. ACE & MWh & 15218 & 4308 & 3224 & 2590 & 2657 & 2630 \\
\hline Sum neg. ACE & MWh & -4097 & -2056 & -1377 & -1335 & -1266 & -1331 \\
\hline $\begin{array}{l}\text { |ACE forecast error } \mid \\
\text { @ last activ. }\end{array}$ & $\mathrm{MWh} / \mathrm{h}$ & & 10.67 & 6.34 & 6.34 & 6.34 & 6.34 \\
\hline Balancing energy & GWh & & 16.419 & 19.116 & 19.394 & 17.527 & 17.605 \\
\hline up-regulation & GWh & & 3.775 & 4.921 & 4.764 & 3.899 & 3.891 \\
\hline down-regulation & GWh & & 12.644 & 14.195 & 14.630 & 13.629 & 13.714 \\
\hline
\end{tabular}




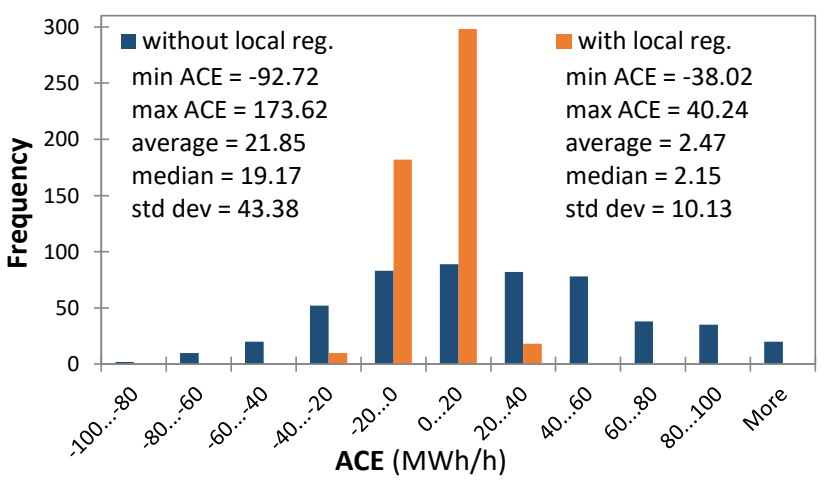

Figure 4. ACE histogram w/o local reg. and w reg. (3 max activations)

Though cost minimization of balancing services was not performed at this stage, it is useful to quantify the economic benefits of common Baltic balancing operations compared to sole reliance on the neighboring Russian power system for balancing needs. If we assume that the OBP buys excess energy at $5 € / \mathrm{MWh}$ and sells deficit at $100 € / \mathrm{MWh}$ [4], then for the test dataset of 509 hours the cost of fully depending on the OBP (cost of the ACE without regulation) would be equal to 333.6 thousand $€$ (76.1 thousand $€$ income from sold energy and 409.7 thousand $€$ expense for purchased energy).

However, when local balancing energy is ordered in accordance with the optimized parameters as presented before, the overall costs decrease notably. For simplicity's sake, the prices of local balancing bids are assumed to be always cleared at $50 € / \mathrm{MWh}$ for upward and $10 € / \mathrm{MWh}$ for downward regulation and perfect liquidity is implied. In all cases with local balancing operations, the total imbalance costs diminish by a factor of 1.35 to 1.94 (depending on the selected max. number of activations) compared to the case with no local regulation. The lowest cost is achieved with 4 and 5 activations (171.9 and 177.4 thousand $€$ respectively). Even with only 1 activation the cost with local regulation is 246.4 thousand $€$ which is significantly lower than without local regulation (333.6 thousand $€$ ).

\section{CONCLUSIONS}

The numerical simulations of balancing activations based on the parameters optimized using historical time series affirmed the generalizability of the results, since in most of the cases the average absolute ACE was close to the forecast error. Additionally, this confirms improved ACE forecasting techniques to be a promising avenue for further research as any enhancements there can be expected to notably improve the efficiency of balancing operations. Furthermore, as currently the balancing parameter optimization tool has a tendency to postpone regulation to later part of the ISP when forecasts are more accurate, perfected early prediction abilities would allow for more even balancing operations throughout the ISP.

For the dataset considered in this study, 4 and 5 were identified as the maximum number of activations during the ISP equal to one hour that can provide the most efficient balancing. While the case with 3 maximum activations did provide a small overall ACE, the aforementioned cases were superior in terms of the utilized balancing energy and estimated cost.

While our tool has yet to be expanded to incorporate the merit order of balancing bids, the initial simplified economic calculations already point to noticeable financial gains from a more active local balancing in the Baltic power system and decreased reliance on regulation from the Russian power system, especially with the OBP's current balancing energy pricing policy.

Even disregarding the financial aspects, the evolution of the common Baltic balancing market is well underway after its launch at the beginning of 2018. Similar trends are ongoing throughout Europe as the TSOs need to adapt the recently established European Commission guidelines on electricity balancing and devise their AOFs for more efficient power system balancing. The work, the beginning of which is presented in this paper, proves to be of significant relevance in the light of the changing balancing market landscape in Europe. The initial results of this study have informed some of the decisions of the Baltic TSOs in the development of their common balancing market. Moreover, this is also important as the Baltic countries strive to desynchronize from the IPS/UPS by 2025 .

\section{REFERENCES}

[1] European Commission, Commission Regulation (EU) 2017/2195 of 23 November 2017 establishing a guideline on electricity balancing. 2017.

[2] "Feasibility study regarding cooperation between the Nordic and the Baltic power systems within the Nordic ENTSO-E pilot project on electricity balancing," 2014. [Online]. Available: http://www.ast.lv/files/ast_files/balansesana/STUDY REPORT-Nordic - Baltic cooperation in Electricity Balancing_112014_final.pdf.

[3] Arturs Purvins; Gianluca Fulli; Catalin Felix Covrig, "The Baltic Power System between East and West Interconnections," 2016.

[4] Pöyry Management Consulting (UK) Ltd, "Baltic's balance management model study and harmonisation plan towards EU energy markets model (including Nordic-Baltic balancing cooperation). A report to Elering," $2016 . \quad$ [Online]. Available: https://elering.ee/sites/default/files/attachments/Pöyry uuring.pdf.

[5] Augstsprieguma tīkls AS, "Annual statement of transmission system operator for the year 2016," 2017. [Online]. Available: http://ast.lv/files/ast_files/gadaparskzinoj/TSO_Annual_Statement_201 6.pdf.

[6] ENTSO-E, "First Edition of the Bidding Zone Review: First Draft," 2018.

[7] M. Haberg and G. Doorman, "A stochastic mixed integer linear programming formulation for the balancing energy activation problem under uncertainty," in 2017 IEEE Manchester PowerTech, 2017, pp. 16.

[8] P. Havel, P. Filas, and J. Fantik, "Simulation-based optimization of ancillary services," in 2008 5th International Conference on the European Electricity Market, 2008, pp. 1-5.

[9] J. Novak, P. Chalupa, and V. Bobal, "Automatic controller for costoptimal power balance control," in 2010 9th International Conference on Environment and Electrical Engineering, 2010, pp. 222-225.

[10] The MathWorks Inc., "MATLAB patternsearch." [Online]. Available: https://www.mathworks.com/help/gads/patternsearch.html.

[11] M. P. Garcia and D. S. Kirschen, "Forecasting system imbalance volumes in competitive electricity markets," in IEEE PES Power Systems Conference and Exposition, 2004, pp. 1115-1122.

[12] S. Lu, Y. V. Makarov, A. J. Brothers, C. A. McKinstry, S. Jin, and J. H. Pease, "Prediction of power system balancing requirement and tail event," in IEEE PES T\&D, 2010, pp. 1-7. 